Bundesgesundheitsbl 2009 · 52:572-572 DOI 10.1007/s00103-009-0864-y

๑) Springer Medizin Verlag 2009

\title{
Maßnahmen zur Vermeidung der transfusionsinduzierten Lungeninsuffizienz (TRALI)
}

\author{
Bei der 67. Sitzung des Arbeitskreises Blut wurde folgendes \\ Votum (V 39) verabschiedet und am 9. Januar 2009 \\ unter www.RKI.de > Infektionsschutz $>$ Blut/Transfusions- \\ medizin > Arbeitskreis Blut > AK Blut Voten veröffentlicht:
}

Die transfusionsassoziierte akute Lungeninsuffizienz ist gekennzeichnet durch ein radiologisch gesichertes, nicht-kardiogenes Lungenödem innerhalb von 6 Stunden nach Transfusion. Die Häufigkeit liegt nach jüngsten Erhebungen in Deutschland bei etwa 1:65.00o für die Transfusion von gefrorenem Frischplasma sowie bei 1:2.266.ooo für Erythrozytenkonzentrate. Ähnliche Größenordnungen werden vom englischen Hämovigilanzsystem (SHOT) angegeben. Die Letalität bei TRALI beträgt nach deutschen, französischen und englischen Hämovigilanzdaten bis zu $20 \%$.

Unter den derzeit diskutierten, potenziell TRALI auslösenden Mediatoren in den transfundierten Blutkomponenten gelten leukozytäre Antikörper, d.h. Antikörper gegen HLA- und HNA-Proteine auf Leukozyten, aufgrund klinischer, tierexperimenteller und in-vitro-Studien als gesicherte Auslöser. Die Auslösung einer TRALI hängt dabei von der Konzentration und Spezifität der Antikörper ab sowie von der Prädisposition des Blutempfängers, eine TRALI-Reaktion zu entwickeln.

Mit Abstand am häufigsten wurden antikörperbedingte TRALI-Reaktionen nach Transfusion von therapeutischem Frischplasma beobachtet, das aus Blutspenden von Frauen mit Geburten in der Anamnese hergestellt wurde.
Zur Reduktion der TRALI-Inzidenz empfiehlt der Arbeitskreis Blut daher, gefrorenes Frischplasma, das von Spenderinnen mit Geburten in der Anamnese stammt, nicht mehr in den Verkehr zu bringen. Plasma von Frauen mit einer oder mehreren Geburten in der Anamnese darf nur dann weiterhin als therapeutisches Frischplasma verwendet werden, wenn es mit einer validierten Methode negativ auf das Vorliegen von leukozytären (AntiHLA-Klasse I, Anti-HLA-Klasse II) und granulozytenspezifischen (Anti-HNA-1a, -1b, -2a, -3a) Antikörpern getestet wurde.

Der Arbeitskreis Blut begrüßt die Einleitung eines entsprechenden Stufenplanverfahrens durch das Paul-Ehrlich-Institut, aus dem die Details und Fristen der Umsetzung zu erwarten sind, insbesondere zur Vermeidung des Verwerfens von bereits hergestelltem gefrorenen Frischplasma.

\section{Für den Arbeitskreis Blut:}

Prof. Dr. R. Burger, Vorsitzender

Dr. R. Offergeld, Geschäftsführerin

Eine englische Fassung der Voten des Arbeitskreises Blut ist verfügbar unter http://www.rki.de $>$ English $>$ Prevention of infection $>$ National Advisory Committee „Blood“. 